\title{
DISORDERS OF PERCEPTION AND PERFORMANCE IN A CASE OF RIGHT-SIDED CEREBRAL THROMBOSIS
}

\author{
BY \\ R. W. GILliatt and R. T. C. PRATt \\ From the Department for Nervous Diseases, the Middlesex Hospital, London
}

The disorders of perception and performance which accompany cerebral injury have a particular fascination in neurology, in that they cannot always be explained solely in terms of the sensory loss or motor deficit observed.

It has been known for some time that in righthanded persons lesions of the right cerebral hemisphere involving the fronto-parietal region may produce, in addition to left hemiplegia and left hemianaesthesia, an agnosia, a failure to recognize the left side, or an anosognosia, a failure to recognize disease of the paralysed side. Typical cases have recently been reported by Sandifer (1946) and by Roth (1949). It has also been shown that right parietal lesions may result in a corresponding neglect of external space on the hemiplegic side (Brain, 1941). There is some doubt as to whether this is a defect characteristic of cerebral lesions on the right side only, or whether it is present but masked by grosser disabilities after left-sided parietal lesions (Brain, 1945). There may also be a failure to solve visual problems and to perform constructional tasks involving spatial analysis and orientation (McFie, Piercy, and Zangwill, 1950). This appears to occur as a relatively clear-cut defect unrelated to any generalized impairment of language, intelligence, or memory.

The object of this paper is to report a case of right-sided cerebral thrombosis followed by left hemiplegia with extensive sensory loss and anosognosia, and accompanied by profound impairment of visual-spatial perception and performance. It was possible to follow the gradual resolution of these abnormalities in some detail, and as there was little, if any, generalized confusion at any stage of the illness, it is felt that certain features emerged of sufficient interest to justify the publication of a single case.

\section{The Onset of the Illness}

Mrs. H., a married woman of 62 , had been well until December 23, 1950 . She was known to have had mild hypertension for 10 years, with a diastolic pressure in the region of $100 \mathrm{~mm}$. Hg. Her pre-morbid intelligence appears to have been high; the wife of a scientist with three grown-up children, she was active, well-read, and interested in her home and the careers of her children. There was no history of any previous cerebral vascular episode. She was right-handed. The following account of the onset of her illness was obtained from her husband.

At 5.30 p.m. on December 23, 1950, Mrs. H. was in the kitchen of her house when she was seen to sway on her feet. She first complained of pins and needles in the left arm, and then that the left leg felt weak, but she was able to walk into another room and sit down. One hour later she was seen by a doctor, who noted some indistinctness of speech and drooping of the left corner of the mouth. The eyes tended to deviate to the right, but the left grip was still "quite good". There was definite weakness of the left leg, and when she insisted on kneeling down to straighten the carpet she was unable to get up without assistance. By 7.30 p.m., when she was helped to bed, the left leg and arm were much weaker, but the patient remained conscious and rational. Her speech had become more indistinct with dribbling of saliva from the left corner of the mouth, and numbness of the left side was first noticed at this time. By the following morning there was profound weakness of the left face, arm, and leg, with loss of feeling, and she complained of severe right frontal headache which persisted for three days. There was no loss of consciousness, and no confusion was noted by her relatives, but little movement of the left arm or leg was seen over the next few days, and she was admitted to the Middlesex Hospital under the care of Dr. D. McAlpine on December 30 with a provisional diagnosis of cerebral thrombosis.

On admission the patient gave an account of her illness which was substantially correct, but there was some confusion as to time and date. She described the onset in the kitchen correctly, and remembered that her daughter had sent her into another room, but whereas she was aware she had lost power in the left leg, she insisted that the left arm had not been affected and that it was normal. Routine questions about her general health, past and family history were answered correctly ; for example, she knew her name, age, home address, and telephone number, and she talked with normal interest of her family and their doings, in particular being con- 
cerned over their reactions to her illness. She also spoke sensibly of her hypertension and of the phenobarbitone which had been prescribed for her in the past.

On general examination signs of mild hypertension were found (B.P. 18595) but no other abnormality outside the central nervous system. A detailed neurological examination was carried out on December 31 , and the findings were as follows :

Speech.--There was no dysphasia. A slight dysarthria was attributed to a weakness of the left side of the face and left half of the tongue.

Mood.-The patient was aware that she was seriously ill and was depressed, but her mood was abnormally labile, and she could be made to laugh or cry easily.

Cooperation.-She cooperated well during examination, but in tests of memory and intelligence she tended to answer rather quickly and would guess rather than think again if she was wrong; this was particularly noticeable when she was tired.

Confusion.- She knew that she was in the Middlesex Hospital and that she had arrived the day before, but was still slightly confused in time. There was difficulty in assessing the number of days that had elapsed since the start of her illness, which she considered to have been on Christmas Day. Otherwise, she showed an almost complete absence of confusion, save in her attitude to her hemiplegia.

Calculation and Retention.--Simple tests with numbers showed that she had a disproportionate difficulty in retaining digits and in adding and subtracting. She could repeat four figures forwards but not backwards. She added $7 \mathrm{~s}$ serially to 8 correctly as far as 36 , but then started adding $9 \mathrm{~s}$, and she was unable to subtract 7 s serially from 100 at all. The Babcock sentence was repeated to her twice, after which her version was: "Thing a nation needs to be rich is a large supply of wood-and food".

Cranial Nerves.-A left homonymous hemianopia was present extending to the fixation point, but acuity for central vision was not grossly reduced. There was no failure of conjugate eye movement to the left but it was poorly sustained. Coarse horizontal nystagmus was present on conjugate deviation to the right. There was complete superficial sensory loss over the left side of the face with absence of the left corneal reflex. Motor power was slightly reduced in the upper facial muscles on the left, with gross lower left facial weakness, and protrusion of the tongue to the left. The head was held slightly turned to the right at rest, and there was some loss of power of rotation to the left. Auditory acuity was normal in both ears. Auditory inattention was not present ; when tested by two examiners speaking on either side of her, she repeated the first figure of a double number spoken into the left ear, and the second figure of a different double number spoken simultaneously into the right ear, thus forming a composite number.

Trunk and Limbs. - Tone and power in the right arm and leg were normal. Voluntary power was absent in the left hand and in all the muscles of the left arm and shoulder, and no contraction of the left trapezius could be obtained. Associated movements were not observed. Tone was slightly increased in the left biceps, the arm being held in partial flexion, but the wrist was still flaccid and deep reflexes were only moderately increased compared with the right.

In the lower limb slight movements of flexion and extension were possible at the left hip, but no other voluntary movement was observed in the leg or foot. Tone was already markedly increased, more at the ankle than at the knee, with correspondingly increased deep reflexes and sustained clonus at the left ankle. A well-marked extensor plantar response was present with flexion of the left knee and hip. The right plantar response was flexor.

There was no appreciation of touch, heat, cold, scratching, or repeated pinpricks over the left side of the neck and trunk to the mid-line, nor over the left arm and leg. Some generalized response to strong painful stimuli was elicited by pinching skin, muscle, or tendon, but did not occur in response to pinprick. (This phenomenon had certain features of interest, and is further described below.) Vibration sensation was lost over the limbs and abdominal wall on the left, and passive movements of the left shoulder and hip were not appreciated. Sensation on the right side remained normal, and sphincter control was unaffected.

\section{Subsequent Progress of Left Hemiplegia}

The recovery of motor power was slow and incomplete. Four weeks after admission slight weakness of the left upper facial muscles was still present, with moderate left lower facial weakness, and the tongue was still protruded to the left. Some power had returned in the left trapezius, but no movemen $i$ at the shoulder joint had been seen. A trace of flexion and extension of the elbow was present but no voluntary movement of the wrist or hand. Tone was markedly increased throughout the arm particularly in the flexors of the elbow, wrist, and fingers, with clonus of the brachio-radialis. There had been slight improvement in power at the left hip, but no voluntary movement at the left knee or ankle. Gross extensor hypertonus was present in the left leg.

By the tenth week the patient had begun to stand, and to walk a few steps supported on each side. Loss of power was complete at the shoulder joint and not less than $80 \%$ at the left elbow, with some flexion but no extension of the wrist and fingers. The strength of the left hip muscles had further improved and was about $50 \%$ normal. Some flexion and extension were possible at the left knee, with a trace of voluntary dorsiflexion at the ankle.

By the twentieth week there had been further improvement in power. There was still no voluntary movement at the left shoulder joint; flexion at the elbow was possible against gravity, but extension only with gravity eliminated. Flexion and extension at the wrist were strong enough to overcome gravity, but no fine movements of the hand were possible. Tone remained grossly increased, particularly in the flexor groups. There had 
been little change in the leg save for the reappearance of a trace of flexion of the toes. Loss of power was estimated as $50 \%$ in the hip muscles and in the quadriceps, and $70 \%$ in the knee flexors. There was gross extensor hypertonus with contracture of the calf muscles.

When she was discharged on June 2, 1951, the patient could walk with a hemiplegic gait using a right elbow crutch and with one person supporting her on the left side.

\section{Sensory Disturbances and Anosognosia}

Agnosia and Anosognosia.-On admission the patient's attitude to her left side was grossly abnormal: when lying in bed she appeared completely unaware of it, and it frequently became uncovered with the leg half out of bed without any attempt being made to replace it or to pull the bedclothes over it. During examination the patient never looked at the left arm or leg, even when asked to concentrate on some movement or during sensory testing. The neglect of her left side obtained even when her head was turned to the left.

On direct questioning she denied that there was anything wrong with the left arm. She admitted to some weakness of the left leg but responded to requests to move it by making the corresponding movements on the right, as with the arm, and in each case would appear satisfied with the result. When the immobile left arm was shown to her, she recognized it as her own, but rationalized her failure to move it, as is shown by her remark, "Can only move it if there is object to move".

Right-left Confusion.-On admission there was difficulty in identifying promptly her own and the examiner's right and left hands. Her performance in this respect was very variable, but even some weeks after admission she was only able to select the examiner's right hand by making a gesture of shaking hands.

Pain Perception.-Initially the patient showed a curious generalized response to strong painful stimuli to the left side. This was only briefly mentioned in the preceding section. It consisted of general restlessness and uneasiness for which the patient could find no cause, and which she never associated with a peripheral stimulus even in repeated tests. The restlessness was accompanied by tachycardia, a rise in blood pressure, dilatation of the pupils, and deepening of respiration, all appearing within one or two seconds of a strong pinch of skin or deeper structures. The response could not at first be elicited by pin-prick. It could be produced from the face, neck, trunk, and limbs on the left side. In the case of the left leg it was accompanied by reflex flexion-withdrawal of the limb, and usually by flexion of the right leg as well.

It was noticed that in addition to the restlessness and movement in bed the patient would sometimes start scratching the chin, neck, or left flank with her right hand. At first this was not an invariable accompaniment of the general response, but later it became more prominent.

Asked if she felt anything, the patient gave replies such as, "Am moving to make me more comfortable", or, when scratching her face, "Chin felt queer". On another occasion she moved restlessly in bed and sighed, and when asked the reason replied : "Not very happy ", adding, "Would you be in my position?" She was quite unaware that her left side was being stimulated and denied pain on every occasion.

\section{Subsequent Progress of Sensory Loss and Anosognosia}

On January 2, 1951, the patient was still mildly confused in time, saying that the day of the week was Monday instead of Tuesday, and although she was corrected was wrong again at the end of the examination. She still maintained that the left arm and leg were " all right" and moved the right instead. Scratching was a prominent part of her response to painful stimuli. Pinching the left ear resulted in scratching of the chin, pinching the arm in scratching of the left side of the neck, and pinching the left leg or foot produced scratching of the left flank. This association was not invariable, and if asked whether she felt anything she would reply that she was " itchy" or " twingey " all over, or rationalize by saying that her flank was sore where she had been lifted and turned in bed. On all occasions she showed general restlessness and uneasiness but denied pain anywhere.

On January 3, when asked about her face, she stated that the left side felt numb "like someone else's face". She said that the left hand was " all right ", but on being asked to show it, showed the right instead. When shown the left hand she recognized it, and after trying to move it said, "Will not lift", and that it was " very naughty". She described the left leg as " all right" but moved the right instead. Painful stimuli to the left side produced restlessness and scratching, as before, without any awareness of the peripheral stimulus. On this occasion there was no general confusion; she named the time and date correctly and remembered the names of her visitors of the evening before.

On January 4 the patient again denied her hemiplegia. On being told to move the left hand, she merely wagged it with the right hand and appeared satisfied with the result; when the hands were separated and another attempt made, she moved the right hand as on previous days. Pin-prick was occasionally felt as dull touch over the upper face, but there was no other change in sensation.

On January 5 the patient's attitude to her hemiplegia was changing but was still grossly abnormal. She tended to make light of her disability, and the following conversation took place :

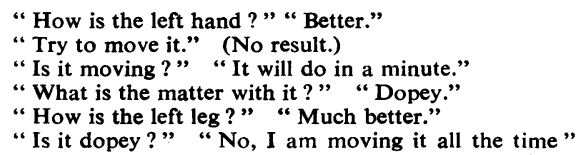

(moving right).

Examination also revealed some improvement in sensation; pin-prick was felt as dull touch over the left face, neck, and arm, but whereas it was correctly lateralized and fairly well localized over the face, in the 
arm it was referred to the right side instead of the left in about half of the replies. This was the first occasion on which allaesthesia was observed. Pin-prick was not perceived as such over the left foot, but produced some scratching of the left neck and clavicle, which the patient described as "twingey". Vigorous pinching of the left big toe produced general restlessness, but on one occasion the patient recognized and localized the stimulus correctly, pointing out the toe with the right hand.

Her lack of general intellectual impairment was shown by a clear and correct account of the nature and scope of her son's work, which she was able to give on this day.

On January 6 further improvement was noted. Asked if she could move the left hand, she said "Yes", but when it was attempted there was no movement. The patient said she did not know why she could not move it, and referred to it as "silly hand". There were some failures to name her own and the examiner's right and left hands correctly and she said, "I confused my right and left, I can't make it out. I'm silly ". To avoid verbal confusion between right and left during sensory testing, the patient was asked to try to point out the site of the stimulus on each occasion. Pin-pricks on the left face were correctly lateralized and were localized to the forehead, cheek, or chin, but were felt as dull touches. Pricking of the arm caused only scratching of the left clavicle. From the left knee a proportion of pin-pricks was felt as dull touches on the left calf, but an equal number was referred to the right knee, or caused withdrawal movements without localized sensation. Hard pinching of the left big toe was similarly either localized, referred to the right toe, or ignored in spite of reflex withdrawal and general body movement.

On January 8 and January 9 the patient still found difficulty in naming the day of the week and the time, and named the examiner's right and left hands wrongly in three consecutive tests. Her attitude to her hemiplegia was one of unconcern, as shown by the following conversation :

\footnotetext{
"How is the left arm ?" "Better."

"Is there anything wrong with it?" " No, I don't think so."

"How is the left leg?" "Better. Ask Nurse" (referring to the physiotherapist).

"Is there anything wrong with it ?" " Don't think so."

" Could you walk on it ?" " Let me try."
}

Pin-prick was usually felt as dull touch and localized to within two to three inches over the face, neck, and flank. Sensory inattention (Critchley, 1949) was present to simultaneous bilateral pin-pricks of approximately equal strength. From the left fore-arm stimuli were referred to the hand, and from the upper arm to the clavicular region. Replies were rather more variable from the leg. Stimuli over the left face and trunk were occasionally referred to the mid-line, but no allaesthesia remained save from a small area on the anterior abdominal wall. Even here it was inconstant, and it was not observed again in subsequent examinations.

The absence of general intellectual impairment may be illustrated by a conversation at this time in which she discussed various books that she had been reading just before her illness (in particular Stefan Zweig's Beware of Pity and a life of Balzac), and bewailed the fact that she had not been able to read since the onset.

By January 16 the disability of the left arm was no longer ignored but was treated in an objective fashion; the patient referred to it as " poor little withered hand ", picked it up, and kissed it. There was still some neglect of the left leg both in bed and when sitting out in the ward. At this time pin-prick was poorly localized and felt as tapping on the left arm and leg, but both pinprick and pinching could still sometimes produce general restlessness. In the case of the arm this was usually accompanied by a "twingey" feeling in the region of the clavicle, and by scratching, but from the leg it could still occasionally be elicited without any localized sensation whatever. This response was not observed again after this date.

On February 13 she still showed considerable emotional lability, becoming very upset over other patients in the ward, and laughing and crying readily. There was no denial or obvious neglect of her hemiplegia, and the patient stated that if she wanted anything from the locker on the left of her bed, she would try to use the left hand without thinking. Pin-prick was felt as touch and correctly localized over the face. It was referred from the left upper arm to the shoulder and interpreted as painful, but was not felt at all on the forearm. On the legs pin-pricks were poorly localized but were painful. Sensory inattention was present. Light touch was not perceived at all over the left side, and postural sensation was absent.

At this time it became apparent that a phantom limb was present. During the early weeks of her illness, on being requested to find the left hand with the eyes closed, the patient had been accustomed to feel for the left shoulder and work her way down the arm to the hand. After the disappearance of anosognosia, if the left arm were held up in the air with the patient's eyes closed, she would feel for the left hand on the lower abdomen " in the usual place" for a few seconds before tracing it by way of her left shoulder. If the left arm were held up in the air and she were asked to place the right arm in the same position as her left arm, she would place it on the abdomen, with fingers separated and extended. She was unable to move the phantom, and readily ignored it when she could see the real position of the left arm. The phantom persisted until her discharge from hospital. The appearance of a phantom with improvement in cerebral function is in interesting contrast to the patient described by Head and Holmes (Head, 1920) in whom a stroke abolished a phantom leg.

On March 18 a considerable degree of sensory loss was still present, but the patient's attitude to her left side was not obviously abnormal, and there was no neglect nor denial of disability. Pin-prick was felt dully over the left face with inattention. There was some blunting over the left arm and forearm, and a pin-prick felt dull on the hand; on the leg it was recognized as painful or "squeezing". It was localized to within three or four inches on both arm and leg. Pinching of the skin was not distinguished from pin-pricks, but was 
recognized as painful. No general restlessness was elicited by either of these stimuli. Postural sensation was absent at the left shoulder but present at the hip. Light touch remained wholly absent over the left face and limbs, and hot and cold stimuli were not appreciated on that side.

On May 16 appreciation of light touch was still absent, but firm pressure was felt and was well localized except on the leg. A prick was recognized by its unpleasant quality and was well localized on both arm and leg. There had been no further improvement in position sense, nor in appreciation of heat and cold.

At a follow-up examination on March 22, 1952, the patient's phantom limb was still present. The phantom hand was felt to be resting on the abdomen with the fingers fully extended, in contrast to the flexed posture of the real fingers. In addition the patient described frequent sensations of individual finger movement although she knew by inspection that these never in fact occurred.

\section{Visual Field Defects}

On admission a left homonymous hemianopia extending to the fixation point was present on confrontation ; the patient denied this even when it was demonstrated to her. One week after admission she was able to appreciate moving objects in the upper part of the left half-fields, with visual inattention, and three days later stationary objects were perceived in the left upper quadrants. Three weeks after admission movements were consistently appreciated in the left lower quadrants but stationary objects were not seen. After one month it was noted that the patient showed no disorientation in the left half-fields, and was able to touch on request the nearer or farther of two objects introduced into them. By the time she was discharged, the patient was able to recognize common objects in the upper but not the lower quadrants, and a partial left lower quadrantic defect was present on perimetry $\left(\frac{1}{2}^{\circ}\right.$ white). Visual inattention was still present.

\section{Unilateral Neglect of External Space}

Drawing.-Shortly after admission a drawing of a clock showed an unmistakable neglect of the left half, all the numbers on the left side being drawn in their mirror-image positions on the right. On January 6 further clock drawings produced an identical result; two days later, however, the patient was able to draw a clock normally, numbering the dial correctly but had great difficulty in drawing in the hands to show a given time.

Reading.-On admission she was able to write her name and address, but her handwriting was almost illegible. She herself had great difficulty in reading. The words "The Middlesex Hospital", printed in capitals at the top of ordinary ward paper, were first read as " hospital", then "Essex hospital", and finally, with encouragement, " The Middlesex Hospital".

On January 6 the patient was tested with the following sentence printed in half-inch block capitals :

\section{GEORGE \\ EATING \\ SUNDAY \\ LIKES \\ HIS \\ DINNER}

This was first read as " likes his dinner ", but on being made to search for the rest of the words, she managed the complete sentence, only to come back to it a few minutes later and read "George likes his dinner", denying the presence of any other words on the page even when it was suggested that she had omitted some.

On January 9, on testing with Snellen's type at six metres, the patient read only the right-hand letter of each of the first five lines. Three days later she read Snellen's type normally (V.A.R. $=6 / 24$, V.A.L. $=6 / 18$ ), but still had great difficulty in reading sentences. She was unable to understand newspapers or letters, as, in attempting to read, she would jump from the end of one line to the middle of the next, and would misread or omit words, syllables, and individual letters, so that even simple sentences became nonsense.

On January 17 it was discovered that her performance was strikingly different when reading from above downwards with the letters in columns starting from the left of the page. The first test sentence is below:

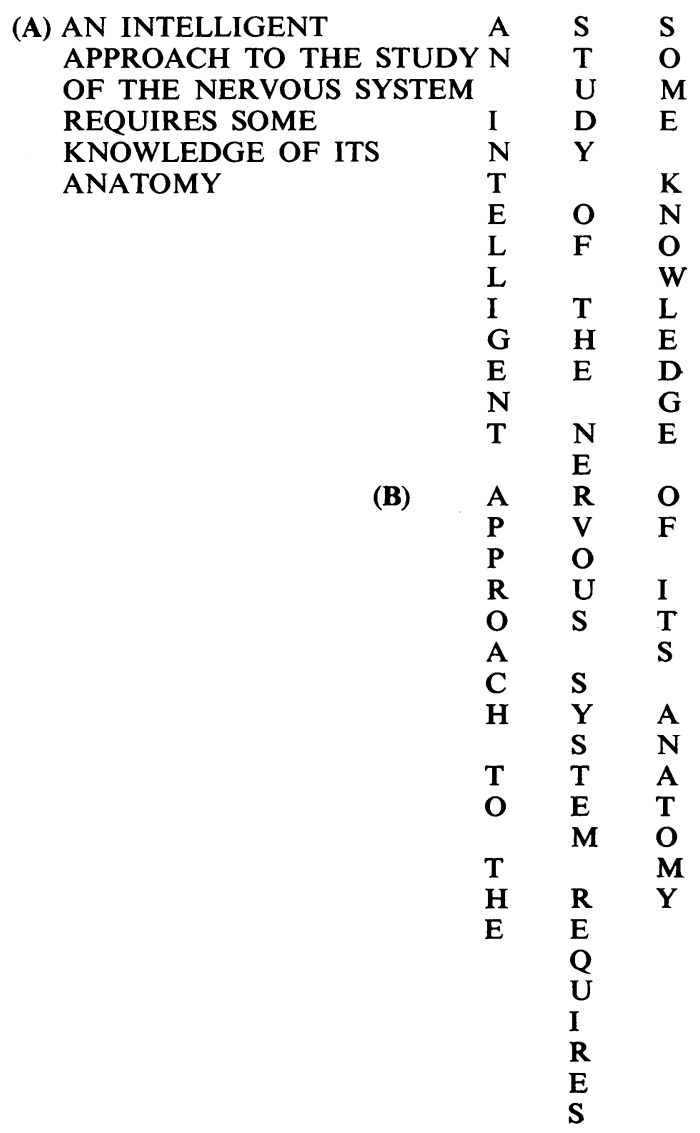


The patient was told to spell out the letters making words where possible. Reading horizontally (A) her version was: "In itellgent enouch to the stry of the blous sytem reuies some boese of its anatmy" (time taken $2 \mathrm{~min} .45 \mathrm{sec}$.). In contrast to this the first time she read the sentence from above downwards (B) she made only two small mistakes: " $\mathrm{Hn}$ intelligemn approach to the study of the nervous system requires some knowledge of its anatomy" (time taken $3 \mathrm{~min}$. $30 \mathrm{sec}$.). This phenomenon was not analysed further, for by January 20 her difficulty in reading horizontally had disappeared and did not recur.

Tactile and Visual Neglect in External Space.-On admission a simple test described by Sendifer (1946) was tried: a number of common objects was spread across the coverlet occupying the whole width of the bed, and the patient was asked to find as many of them as possible by groping with the right hand with the eyes shut. She searched approximately equally to her right and left, and recovered all the objects save one on the extreme left at the limit of her reach. In a further test one week later she recovered all the objects without an omission.

On January 11 an attempt was made to investigate the discrepancy between her visual and tactile performance in the left half of space. The patient was presented with a large board covered with paper in which a number of drawing pins was placed at random. The patient was then asked to ring each pin with the right hand, using different coloured pencils with the eyes open and shut respectively. With the eyes open she started in the middle of the board and ringed only those pins to the right, saying at the end, "That's the lot", and denying that she had omitted any. With the eyes shut she missed occasional pins indiscriminately, but worked gradually over to the extreme left of the board, and finished by running her fingers down the left-hand edge. In a subsequent test she was restarted on the extreme left with her eyes open and told to ring those that she had missed, but even with this encouragement she only found a few pins before drifting back to the centre of the board and ignoring those remaining to her left. It was not until February 1 that this test was performed normally with the eyes open.

\section{Visual-spatial Agnosia}

The patient showed disorders of visual-spatial perception of the type analysed by McFie, Piercy, and Zangwill (1950) and referred to by them as visualspatial agnosia. Initially there was no difficulty in revisualizing people or places nor in recognizing common objects, and distances were judged approximately correctly. She made no errors in testing for deformation of coordinates, nor in bisecting lines. It was not possible to demonstrate any gross defect of route finding in her new surroundings. Topographical memory was not impaired, and she was able to describe her route home from the hospital with detail and accuracy. Similarly, she could describe the main turnings off Piccadilly Circus correctly, and mention a number of well-known buildings and shops in the vicinity. In contrast to this she was incapable of the simplest plan drawing. In drawing Piccadilly Circus, for example, she would start with Oxford Street drawn vertically down the paper, and add everything else in a haphazard jumble to the right of it. Her copying of simple geometrical patterns was very faulty, and drawings of objects from memory showed marked dysjunction.

Three weeks after admission, on the Progressive Matrices (1938) Test, she chose only eight correct patterns out of the 12 in Set A. Six weeks after admission, when tested with Kohs Blocks (Wechsler Form 2) she was unable to proceed beyond the demonstration pattern, even when shown the solution to pattern 1 . When re-tested on progressive matrices just before her discharge from hospital, she showed little improvement, with only 14 correct choices out of the 24 in Sets A and B, whereas a vocabulary test (Wechsler) gave an intelligence quotient of 119.

Owing to her hemiplegia it was not possible to perform any satisfactory test of apraxia for dressing.

\section{The Relation of Unilateral Neglect of External Space to Visual-spatial Agnosia}

Attempts were made to assess the extent to which the patient's neglect of the left half of visual space was contributing to her general difficulty in dealing with visual-spatial problems. Two tests in particular were used and merit brief description.

On January 8 it was noted that she was able to draw a clock normally, but had great difficulty in drawing in the hands. When this was investigated further with a large cardboard clock, it did not appear to be related to a neglect of the left half of the clock, as her performance was no better when setting the hands or telling the time on the right half of the clock face. She appeared to realize her disproportionate difficulty, saying on one occasion, "Fancy not being able to do simple stuff like this ", and adding that she did not seem to be able to think what it should look like. The patient had equal difficulty in solving clock problems in her head, and would be defeated by a question such as, "If the hour hand is at 3 , and the minute hand at 10 , what is the time?" This was not thought to be part of a dyscalculia, as she was able to add and subtract 5 s serially to and from 60 with ease. Her performance in clock problems improved only slowly, and mistakes were still made in decreasing numbers until the third month of her stay in hospital.

A second test, suggested by Dr. Michael Kremer, was devised with a simple circular maze 18 in. in diameter, the turnings being so arranged that in finding a way from the centre to the outside, there was a short way involving left turns and a long way using right turns (Fig. 1). It was hoped that this might reveal a defect in route-finding due to neglect of the left turnings, and by making the maze of thick circular strips of orthopaedic felt stuck on a flat board it was possible to compare the patient's performance using sight and touch. This maze was first used on January 15 , when tests with drawing pins and in reading still indicated some visual 


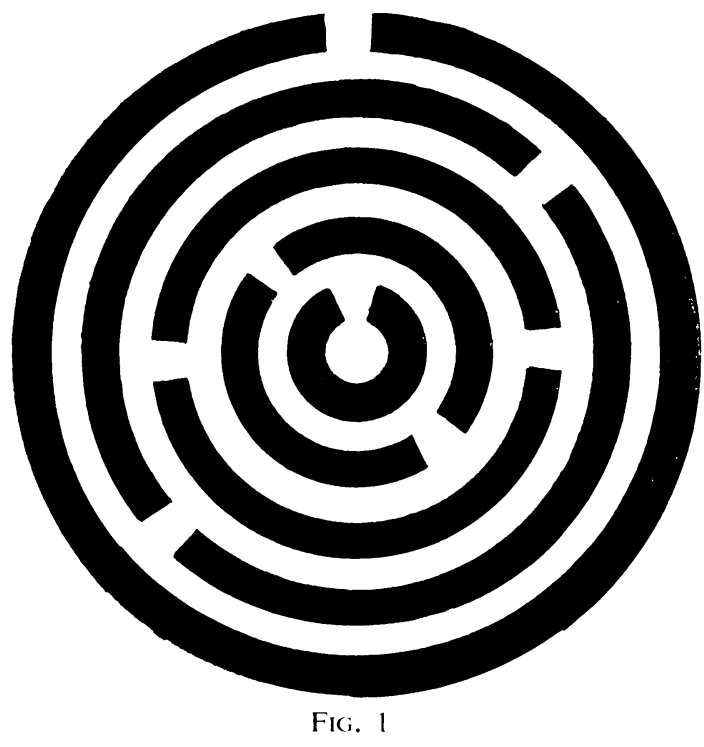

neglect of the left, but it showed in fact not a neglect of turnings to the left, but a complete inability to select turnings leading outwards rather than those leading back to the centre. Although the nature of the problem was well understood, the patient always took random turnings, and would sometimes return to the centre as many as three times before reaching the outside. By touch she was only slightly better than with the eyes open, and with both she appeared to be incapable of pausing to consider any one turning in relation to the whole problem. Her performance improved only slowly, and in spite of repeated use of the same maze it was still abnormal two months later.

\section{Discussion}

On clinical grounds there seems to be little doubt that this patient suffered a cerebral thrombosis resulting in damage to a large area of the right cerebral hemisphere. Consideration of a more exact anatomical localization is not profitable in the absence of necropsy material. An electroencephalogram taken shortly after admission was indicative of a right-sided lesion. It showed frequent replacement of the alpha rhythm in the right occipital area by low voltage $7 / \mathrm{sec}$. waves with high voltage $2-3 / \mathrm{sec}$. waves in the right parietal and frontal areas. Seven weeks later $1-2 /$ sec. waves were still present in the posterior part of the right temporal region and in the right occipital region; further follow-up E.E.G.s showed the alpha rhythm returning to those areas in which it had previously been suppressed.

The anosognosia showed certain points of interest. The denial of disability was focal, including the hemianopia and left arm, but not extending fully to the left leg. It was not associated with any clouding of consciousness nor with generalized confusion. The patient's acceptance of her disability was initially qualified by attempts to rationalize her failure to move, and was later characterized by a curious objective attitude, as if her hand were leading a perverse, independent existence for which she was not directly responsible.

Against the background of profound unilateral loss of pain perception, the patient showed restlessness and uneasiness, tachycardia, rise in blood pressure, pupillary dilatation, and deepening of respiration in response to noxious stimuli on the left side, a phenomenon which slowly vanished in rough correspondence to the anosognosia. Four stages in this process could be discerned. Initially general restlessness and uneasiness were unaccompanied by any recognition of the stimulus. Later the general response was accompanied by scratching of the left side, usually the chin, neck or flank, in response to stimulation of the ear, arm, or leg respectively, and later still the peripheral stimulus began to be fairly consistently interpreted as itching or twinges in these areas. Finally the patient began to recognize and localize the stimulus in a large proportion of tests, the generalized response still appearing occasionally without local sign. This phenomenon has received scant attention in the literature of anosognosia. A similar instance has been described by Pötzl (1924, Case 2). Generalized movements with denial of pain have also been noted by Nathan (personal communication) in patients with anosognosia receiving injections of penicillin on the affected side. Our case differs in certain particulars from Sandifer's (1946) description of a patient in whom noxious stimuli "evoked every expression of pain but denial that she felt any". Allaesthesia in our patient seemed to be a transient phenomenon which appeared at an early stage of sensory recovery and lasted only a few days.

The patient's initial dyslexia may be ascribed to a massive neglect of the left half of visual space, but the discrepancy between her performance in horizontal and vertical reading is more difficult to analyze. It is likely that vertical reading was accomplished by identification of single letters and subsequent synthesis into words, this latter being performed correctly but slowly, indicating some defect in conceptual thinking where visual symbols were involved. The reason for her horizontal dyslexia remains obscure. It may have been due to a partial neglect of the left half, although by this time she was able to find correctly the beginning of each new line of print. Gross oculomotor defects were by this time absent, movements being full in 
all directions and unaccompanied by nystagmus. Neglect of the left side may be sufficient to upset saccadic eye movements in horizontal reading more than in vertical reading, and may have been responsible for her poor , performance (Zangwill, personal communication). On the hypothesis that letters in the right half of the visual field might be causing visual inattention to the left half, an attempt was made to cause inattention during vertical reading by interpolated visual stimuli in the right halffields; no deterioration in performance resulted from this manoeuvre, but this negative result may not be significant.

In general, visual-spatial disturbances outlasted the body image disturbances, but this may merely indicate that the cerebral damage was most severe in the posterior part of the right hemisphere. The disorders of visual-spatial perception conformed to those described by McFie and others (1950). The intactness of topographical orientation and memory in this patient is in accord with the findings of Hécaen, Ajuriaguerra, and Massonnet (1951) in six patients with parieto-occipital lesions of the minor hemisphere, and contrasts with those of McFie and others (1950), who recorded such defects in six of their eight patients.

Neglect of the left half of external space was shown on the motor side in drawing numbers on a clock face, and on the sensory side in reading tests. However, when the patient was required to ring drawing-pins on a board with the eyes open and closed, her performance was strikingly better with the eyes closed. It thus appears that in this patient a neglect of the left half of space was determined by visual experience, and that external space (Brain, 1941) may be further divisible into "visual space" and "tactile space".

Two further problems remained largely unsolved by our analysis, the first of these being the extent to which unilateral neglect of visual space contributed to visual-spatial agnosia. All that can be inferred from our patient is that gross neglect of the left improved relatively early, with persistence of visualspatial agnosia. However, the continued tendency to put in towns on the right side of a map when map-drawing (which was still evident on February 15) indicated some persistent neglect of the left although grosser tests failed to reveal it. The extent to which conceptual processes involving visual symbols were affected in our patient also proved difficult to assess. Her failures to do clock problems in her head, and to do the maze test with the eyes shut were similar to her performance when doing these tests by sight. Therefore, in spite of her denial of difficulty in revisualizing people or places, it is hard to resist the inference that a conceptual defect was present where visual problems were involved. It would be of some interest to know whether her visual concepts lacked a left half, and if so, whether this was an inattention which could have been modified by extraneous visual stimuli.

\section{Summary}

A right-sided cerebral thrombosis in a woman aged 62 gave rise to a left-sided hemiplegia and hemianopia, with profound left-sided sensory loss.

An initial anosognosia was followed by a detached attitude to her disability.

During the stage of profound left-sided sensory loss noxious stimuli to the left side gave rise to restlessness and uneasiness, with tachycardia, rise in blood pressure, pupillary dilatation, and deepening of respiration but without any conscious recognition of the stimulus. The stages in the resolution of the phenomenon were followed.

There was a neglect of the left half of space which was demonstrable only when the eyes were open.

During the stage of recovery vertical reading was performed better than horizontal reading.

Visual-spatial agnosia was a marked feature of the illness, and outlasted neglect of the left half of space.

We wish to thank Dr. Douglas McAlpine for his help and for permission to publish this case, and Dr. Michael Kremer for his interest and advice on the problems presented by the patient.

Brain, W. R. (1941). Brain, 64, 244.

\section{REFERENCES}

B(1945). Lancet, 2, 837.

Critchley, M. (1949). Brain, 72, 538.

Head, H. (1920). Studies in Neurology, vol. 2, p. 606. London. Hécaen, H., Ajuriaguerra, J. de, and Massonnet, J. (1951). Encéphale, 40, 122 .

McFie, J., Piercy, M. F., and Zangwill, O. L. (1950). Brain, 73, 167.

Pötzl, O. (1924). Z. ges. Neurol. Psychiat., (Orig.) 93, 117.

Roth, M. (1949). Brain, 72, 89.

Roth, M. (1949). Brain, 72, 89.
Sandifer, P. H. (1946). Ibid., 69, 122. 